\title{
La notion de dommage exceptionnel comme base pour la réparation du préjudice subi par les femmes exerçant le petit commerce informel durant la période de l'état d'urgence sanitaire dans la ville de Kinshasa
}

\author{
Par Genèse BIBI EKOMENE*
}

\section{Résumé}

Cette étude est un plaidoyer en faveur des femmes exerçant le petit commerce informel et qui ont été durement frappées par les mesures d'état d'urgence édictées pour la lutte contre la propagation du Coronavirus de décembre 2019 (COVID-19). L'auteure va explorer les voies de solutions possibles pour rétablir les femmes victimes dans leurs droits touts au moins d'avant la COVID-19. Les deux premières voies sont gouvernementales. Il s'agit d'une part, de la batterie des mesures économiques pour atténuer les effets néfastes de la COVID-19 décidées par le Gouvernement. Parmi ces mesures, on trouve les exonérations et les allégements fiscaux. D'autre part, il s'agit du Fonds national de solidarité contre le coronavirus (FNSCC) dont la mission est notamment de soutenir les personnes exerçant une activité économique anéantie par les conséquences néfastes de la pandémie. Cependant, la première hypothèse ne concerne pas les personnes exerçant le petit commerce informel qui ont perdu tous les revenus suite à l'arrêt de leurs activités et la deuxième voie celle du FNSCC est inefficace. Seule la voie judiciaire pourrait consister en la saisine du Conseil d'Etat pour dommage exceptionnel en vue d'obtenir réparation telle que prévu par les dispositions légales en vigueur.

\begin{abstract}
This paper is an advocacy for women engaged in informal small-scale trade who have been hard hit by the state of emergency measures enacted to control the spread of the Coronavirus of December 2019 (COVID-19). The author will explore possible solutions to restore the rights of women victims at least before COVID-19. The first two paths are governmental. On the one hand, the battery of economic measures to mitigate the harmful effects of COVID-19 decided by the Government. These measures include tax exemptions and tax breaks. On the other hand, there is the National Solidarity Fund against Coronavirus
\end{abstract}

* Genèse BIBI EKOMENE, Apprenante en vue de l'obtention du Diplôme d'Etudes Supérieures (DES) en Droit à l'Université de Kinshasa. Chercheure et assistante à la coordination des projets du Centre de Recherches et d'Etudes sur l'Etat de Droit en Afrique (CREEDA). Membre du CODESRIA. Email : geneseb@creeda-rdc.org, genesebibiekomene@gmail.com; tél. : (+243) 817660091 , 844502924. 
(FNSCC), whose mission is to support people whose economic activity has been destroyed by the adverse consequences of the pandemic. However, the first hypothesis does not concern people engaged in small-scale informal trade who have lost all their income due to the cessation of their activities and the second path that of the FNSCC, is ineffective. Only the judicial channel could consist in referring the matter to the Council of State for exceptional damage in order to obtain compensation as provided for by the legal provisions in force.

\section{INTRODUCTION}

L'humanité est aujourd'hui confrontée à une crise sanitaire qui semble contraster le progrès scientifique enregistré dans le domaine de la médecine. Il s'agit de la pandémie de coronavirus de décembre 2019 (COVID-19). Cette maladie a affecté 90 millions des personnes au monde causant près de 2 millions des décès en ce début du 2021, soit une année seulement après le déclenchement de cette pandémie dans la ville de Wuhan (Chine). ${ }^{1}$ Outre le bilan humain, la pandémie à COVID-19 a engendré une crise à la fois économique et sociale ${ }^{2}$ qui a, en un laps de temps, amoindris les efforts consentis pour la réduction de la pauvreté en faveur des personnes vulnérables dont les femmes.

A ce sujet, un rapport d'une organisation non gouvernementale (Oxfam- France) décrit les conséquences de la Covid-19 sur les femmes de la manière suivante :

Si la crise sanitaire a touché le monde entier, les inégalités face à ses impacts sont considérables. Les femmes, déjà en première ligne de l'épidémie, sont aussi les premières impactées par les conséquences économiques de la crise. Sur-représentées dans les métiers les plus précaires et les moins bien rémunérés, elles ont été touchées de plein fouet par la pandémie. Pour les mères célibataires, la situation est d'autant plus préoccupante qu'elles sont plus nombreuses à travailler en temps partiel subi (deux fois plus que les femmes en couple). Les mères isolées sont le type de ménage le plus fréquemment rencontré dans les accueils du Secours Catholique selon leur dernier rapport ${ }^{3}$.

La République Démocratique du Congo (RDC) a connu ses premiers cas des personnes contaminées par la COVID-19 le 10 mars 2020 et face à la propagation rapide, le Président de la République a décrété, le 19 mars 2020, l'état d'urgence sanitaire comprenant des mesures restrictives des droits et libertés des personnes notamment l'interdiction de tous les

1 AFP \& TV5 Monde, " Coronavirus : ce que révèlent les taux de mortalité par pays et par continent », disponible sur https://information.tv5monde.com/info/coronavirus-ce-que-revelent-les-tauxde-mortalite, (Consulté le 30 janvier 2021).

2 Voir le rapport du Gouvernement congolais intitulé « Impacts sanitaires et socioéconomiques de la COVID-19 en République Démocratique du Congo. Analyse prospective et orientations de la riposte multisectorielle », Kinshasa, mai 2020.

3 https://www.oxfamfrance.org/inegalites-et-justice-fiscale/inegalites-et-pauvrete-en-france-les-voyan ts-au-rouge/, (Consulté le 12 décembre 2020). 
rassemblements, les réunions ainsi que la fermeture momentanée des écoles, de églises, des bars et des restaurants. ${ }^{4}$

Ces mesures ont eu des effets néfastes notamment sur le petit commerce informel des femmes dans la mesure où elles ne pouvaient plus écouler leurs produits à cause de restriction de mouvement de la population ou le confinement de la Commune de la Gombe. Il importe de signaler que ces activités commerciales de ces femmes se déroulent dans les lieux considérés par les autorités gouvernementales comme propice pour la propagation rapide de la Covid-19. Durant trois mois (mars à juin 2020), certaines femmes pratiquant le commerce informel ont, non seulement arrêté leurs activités économiques, mais aussi consommé les fonds de leur capital commercial. Une situation qui a eu des conséquences sur les équilibres familiaux et sur le processus d'atteinte de l'Objectif de Développement Durable (ODD) relatif à l'autonomisation des femmes ${ }^{5}$.

Pour remédier à cette situation des femmes ayant perdu toutes leurs activités économiques à la suite des mesures de riposte contre la COVID-19, cette étude a exploré les pistes de solutions juridiques en vue de rétablir ces femmes dans leurs droits, du moins d'avant mars 2020. La première piste est celle des mesures économiques pour atténuer les effets néfastes de la Covid-19. La deuxième voie est celle du Fonds national de solidarité contre le coronavirus (FNSCC) dont la mission est notamment de soutenir les personnes exerçant une activité économique anéantie par les conséquences néfastes de la pandémie. La troisième solution est la voie judiciaire. Dans cette perspective, les conséquences subies par ces femmes du fait des mesures de lutte contre la Covid-19 seront considérées comme un dommage exceptionnel et qui nécessite réparation de la part du Gouvernement.

Pour ce faire, cette étude examine deux points. Le premier point fait l'état des lieux des mesures d'urgences et leurs conséquences sur le petit commerce informel exercé par les femmes dans la ville de Kinshasa. Le deuxième examine l'efficacité et l'efficience de pistes de solutions susmentionnées dans la quête relative à la réparation du préjudice subi par les femmes œuvrant dans le secteur économique informel à Kinshasa.

4 Article $1^{\text {er }}$ de l'Ordonnance $\mathrm{n}^{\circ} 20 / 014$ du 24 mars 2020 portant proclamation de l'état d'urgence sanitaire pour faire face à l'épidémie de Covid-19, Journal Officiel de la République Démocratique du Congo (JORDC), $61^{\text {ème }}$ année, Numéro spécial du 16 avril 2020.

5 Lire Genèse Bibi Ekomene, La promotion de l'entrepreneuriat féminin par le microcrédit en République Démocratique du Congo, Librairie africaine d'études juridiques, pp. 350-361, disponible sur https://www.nomos-elibrary.de/10.5771/2363-6262-2020-2-350/la-promotion-de-1-entrepreneuriat-f eminin-par-le-microcredit-en-republique-democratique-du-congo-volume-7-2020-issue-2? page $=1$, consulté le 11 février 2021. 


\section{A. MESURES D'URGENCE ET LEURS CONSEQUENCES SUR LE PETIT COMMERCE INFORMEL EXERCE PAR LES FEMMES DANS LA VILLE DE KINSHASA}

Il est question ici d'analyser les différentes mesures d'urgences prises le Président de la République en vue de limiter la propagation de la COVID-19 (I.) et de mettre en exergue les conséquences de celles-ci sur les activités exercées par les femmes dans l'informel (II.).

\section{Mesures de restriction édictées dans le cadre de la riposte contre la COVID-19}

Le Président de la République a décrété « l'état d'urgence » sur l'ensemble du territoire national ainsi que l'isolement de la ville de Kinshasa du reste du pays afin d'éviter la propagation du coronavirus hors de la capitale, où il reste pour l'instant cantonné6. Il faut noter que la propagation a provoqué l'interruption du fonctionnement régulier des institutions de la République et est susceptible de causer une catastrophe sanitaire mettant en péril, par sa nature et sa gravité, la santé de la population ${ }^{7}$. D'où la nécessité de prendre des mesures urgentes. Ces mesures ${ }^{8}$ sont entre autres :

- La fermeture de toutes les frontières du pays aux passagers et à toute personne, sauf pour les navires cargos et autres moyens de transport frets qui sont autorisés à accéder au territoire national. Leurs personnels sont cependant soumis aux contrôles de santé publique nécessaires;

- L'interdiction de tous les voyages de la capitale vers les Provinces et vice-versa, afin de permettre le confinement de la Ville de Kinshasa, foyer de la pandémie. Pour ce faire, chaque Responsable d'Institutions ou des Services est chargé de prendre des mesures de service minimum pour palier le confinement, à leurs domiciles des autres agents de l’État;

- L'interdiction de tous rassemblements, réunions et célébrations de plus de vingt (20) personnes sur les voies et lieux publics en dehors du domicile familial, la population étant priée de rester à domicile et de n'effectuer que les déplacements strictement indispensables aux besoins professionnels, familiaux ou de santé;

- L'interdiction de tous les mouvements migratoires, par les transports en commun, des bus, camions et autres véhicules de l'intérieur vers la capitale et de la capitale vers l'intérieur. A cet effet, des barrières seront érigées par les Gouverneurs de Provinces et les équipages se soumettront au contrôle de rigueur en matière du Coronavirus;

- L'interdiction de tous les transports fluviaux des passagers de Kinshasa vers les Provinces et vice-versa. Seuls les bateaux et les embarcations transportant les marchandises avec équipages et convoyeurs seront autorisés;

6 https://www.jeuneafrique.com/915794/politique/coronavirus-en-rdc-felix-tshisekedi-decrete-letat-d urgence-et-isole-kinshasa, (Consulté le 03 décembre 2020).

7 Préambule de l'Ordonnance, note 5.

8 Articles 2 et 3 de l'Ordonnance, note 5 . 
- La fermeture provisoire sur toute l'étendue du territoire national des écoles, des universités, des instituts supérieurs officiels et privés et tous établissements recevant du public ainsi que des lieux de réunion, avec effet à la date du 19 mars 2020 pour une durée de quatre (4) semaines;

- L'interdiction de l'ouverture des discothèques, bars, cafés, terrasses et restaurants ainsi que l'organisation des deuils dans les salles, les domiciles ou sur la voie publique, les dépouilles mortelles devant être conduites directement de la morgue jusqu'au lieu d'inhumation et en nombre restreint d'accompagnateurs; et

- La suspension de tous les cultes religieux pour une période de trente (30) jours prenant effet à la date du 19 mars 2020 ainsi que toutes les activités sportives dans les stades et autres lieux de regroupement sportif.

Telles sont les mesures d'urgence prises par le Président de la République en vue de limiter la propagation de cette pandémie. Ces mesures étant bonnes du point de vue sanitaire, celles-ci ont par contre causé des conséquences négatives sur l'économie congolaise en général et le petit commerce informel exercées par les femmes dans la ville de Kinshasa foyer de cette pandémie, en particulier.

\section{Conséquences des mesures de restriction édictées dans le cadre de la riposte contre la COVID-19}

Le petit commerce informel des femmes est devenu un moyen incontestable auquel plusieurs femmes congolaises recourent pour couvrir les besoins quotidiens de leurs ménages. Dans la plupart de cas, ces femmes sont dans une situation sociale de vulnérabilité. Elles sont soit des filles-mères, des femmes seules, des femmes divorcées ou en séparation des corps, des veuves et des femmes mariées. Pour cette dernière catégorie, elles viennent au secours de leurs maris qui pour les uns sont chômeurs et les autres dont leurs salaires n'arrivent pas à répondre à tous les besoins du foyer' ${ }^{9}$.

La période d'avant la pandémie, ces femmes exerçaient librement leurs activités chacune dans sa spécialité. Grâce au bénéfice généré par celles-ci, la vie de leurs foyers étaient équilibrées. Elles assuraient les frais scolaires et académiques des enfants, le paiement de loyer pour ceux qui sont locataires, la prise en charge de la nourriture et des soins médicaux et autres besoins du ménage. Pour certaines, elles pouvaient même supporter d'autres charges des membres de la famille ${ }^{10}$.

9 Lire aussi, Milaine Rossanaly, « À la découverte des femmes entrepreneurs de RDC : entre nécessité et ambition », https://www.banquemondiale.org/fr/news/feature/, (Consulté le 30 janvier 2021).

10 Genèse Bibi Ekomene, Renia Binaki Bamangana \&Yvette Mutunda Tshibuila, Impact du covid-19 sur les femmes exerçant le petit commerce informel dans la ville de Kinshasa (RDC). Cas de madame claire $\mathrm{x}$, vendeuse de pains et arachides, https://www.creeda-rdc.org/resources/documents/cr eeda-covid.pdf, (Consulté, le 30 janvier 2021). 
Depuis l'avènement de la COVID-19 et l'entrée en vigueur des mesures de riposte, la vie de la plupart des femmes exerçant le petit commerce informel est devenue de plus en plus difficile. La plupart de ces femmes vendent dans les milieux environnant les écoles, les églises, les terrains de football et autres coins populaires ou points chauds de la ville de Kinshasa. La fermeture momentanée de ces endroits, l'interdiction de regroupement de plus de vingt personnes et des funérailles ont occasionné la diminution voire la cessation de vente des marchandises de ces femmes. Le confinement qui condamne chacun de rester chez soi, sauf en cas de nécessité, a eu comme conséquence à Kinshasa la baisse de circulation des personnes ${ }^{11}$. Ce qui fait que la marchandise qui pouvait se vendre en une journée passe de deux à trois jours ${ }^{12}$.

Pour madame Pauline ${ }^{13}$ par exemple, vendeuse de bananes plantains, arachides et oranges affirme être victime des mesures d'état d'urgence sanitaire. Durant les trois mois de l'état d'urgence, elle n'enregistrait que les invendus chaque soir. Sa marchandise étant périssable et n'ayant pas de moyens pour une bonne conservation, elle assistait impuissante face au pourrissement d'une bonne quantité de sa marchandise et ce, chaque jour pendant au moins quatre jours en attendant que le premier lot ne finisse pour acheter un autre. C'est pareil pour d'autres femmes vendeuses de pains, farine et autres produits de consommation courante ${ }^{14}$.

Outres ces mesures, il y a également la fermeture des frontières, l'interdiction des mouvements entre Kinshasa et d'autres provinces. Bien que cette mesure laisse l'accès aux biens de première nécessité, celle-ci a occasionné quelques perturbations, notamment la rareté de la marchandise. Cette rareté est dû au fait que les véhicules transportant les marchandises connaissent des nombreuses tracasseries en route et prennent beaucoup de jours plus que prévu pour arriver à destination ${ }^{15}$. Ce qui fait que certains biens n'étant pas bien conservés ou ayant épuisé le délai de conservation, périssent en route.

Eu égard de ce qui précède, cette situation a conduit certaines femmes à la cessation de vente dû au manque de fond commercial. L'effondrement du capital social a pour conséquence au niveau des femmes exerçant le petit commerce informel l'aggravation de l'état de la pauvreté qui se traduit par des journées sans nourriture adéquate pour les enfants, le difficile accès aux soins de santé primaires et le déménagement provoqué par le manque de

11 Juliette Dubois, « En RDC, l'activité tourne au ralenti et la pauvreté gagne du terrain à Kinshasa », https://www.lemonde.fr/afrique/article/2020/10/29/, (Consulté le 30 janvier 2021).

12 Genèse Bibi Ekomene, Renia Binaki Bamangana \& Yvette Mutunda Tshibuila, note 11.

13 Genèse Bibi Ekomene, Renia Binaki Bamangana et Yvette Mutunda Tshibuila, Impact du covid-19 sur les femmes exerçant le petit commerce informel dans la ville de Kinshasa (RDC). Cas de madame Pauline Omoni vendeuse des fruits, Bananes plantains et arachides, disponible sur www.cree da-rdc.org.

14 Lire les monographies, disponibles sur www.creeda-rdc.org.

15 Genèse Bibi Ekomene, Renia Binaki Bamangana \&Yvette Mutunda Tshibuila, Impact du covid-19 sur les femmes exercant le petit commerce informel dans la ville de Kinshasa (RDC). Cas de madame Mamie Luzayadio vendeuse de la farine de manioc, https://www.creeda-rdc.org/resources/do cuments/creeda-covid.pdf, (Consulté, le 30 janvier 2021). 
l'argent pour payer le loyer (bien que le Gouvernement ait demandé aux bailleurs de ne pas tracasser les locataires durant la période d'état d'urgence). La situation de ces femmes doit être analysée comme un cas de violation des droits de l'homme et dont la réparation nécessitera une intervention des pouvoirs publics.

De façon générale, ces familles sont dans une situation très difficile en espérant trouver un appui du Gouvernement afin de reconstituer leur capital social. La question cruciale qui se pose, du point de vue juridique, est celle de savoir par quelle voie ces femmes pourrontelles obtenir cet appui? La réponse à cette question est l'objet de notre réflexion au point II.

\section{B. ANALYSE DES PISTES DE SOLUTIONS POUR L'AMELIORATION DE LA SITUATION DES FEMMES EXERCANT LE PETIT COMMERCE INFORMEL A KINSHASA}

Il est question ici d'explorer les voies de solutions possibles pour rétablir les femmes victimes dans leurs droits tout au moins d'avant la COVID-19. Les deux premières pistes de solution sont gouvernementales. Il s'agit d'une part, de la batterie des mesures économiques pour atténuer les effets néfastes de la COVID-19 décidées par le Gouvernement (I.) et d'autre part du Fonds national de solidarité contre le coronavirus (FNSCC) (II.). La troisième et dernière solution est l'action judiciaire (III.) en vue de la réparation du préjudice subi par ces femmes.

\section{Limite des mesures d'atténuations des effets néfastes de la COVID-19 : exclusion des femmes exerçant le petit commerce informel}

L'état d'urgence a pour objectif, par sa nature, de restreindre les droits et libertés fondamentaux des individus reconnus par les textes en vigueur, pour sauvegarder l'intérêt général. Les mesures édictées par le Président de la République ont été prises dans ce contexte tout en sachant qu'elles devaient avoir des impacts négatifs dans certains ménages dans des grandes villes et plus particulièrement à Kinshasa. Conscient de cette réalité, les autorités gouvernementales ont édictées des mesures alternatives en vue d'atténuer ces impacts. En mai 2020, le Gouvernement a pris des mesures économiques en vue d'atténuer les effets néfastes de la COVID-19. Il s'agit de la mise en place des modes appropriés d'approvisionnement des villes en denrées alimentaires pour prévenir toute rupture de stock afin de mettre la nation à l'abri de toutes conséquences désastreuses sur le plan de la sécurité alimentaire, et des troubles éventuels à l'ordre public ${ }^{16}$. Ainsi que le Gouvernement central a pris des mesures tendant à soutenir l'offre des produits de première nécessité, à faire appliquer des mesures de politique budgétaire et des mesures de politique monétaire, de change et elles visent le secteur financier.

16 Article 6 de l’Ordonnance $\mathrm{n}^{\circ}$ 20/014 du 24 mars 2020, note 5. 
Il s'agit « des mesures d'atténuation des effets néfastes du COVID 19 sur l'économie congolaise qui s'est soldée ce jour par la validation de ces décisions ${ }^{17}$. Ces mesures concernent l'exonération de tous les impôts droits, taxes et redevances sur l'importation et la vente des intrants et produits pharmaceutiques ainsi que sur les matériels et équipements médicaux liés à la riposte au Coronavirus pour une période de 6 mois, l'enlèvement d'urgence des intrants et produits pharmaceutiques actuellement sous douane et la suspension durant 3 mois des paiements de l'impôt sur le revenu locatif à charge des entreprises...(le) soutien pour la relance des activités entreprises au moyen d'un financement du Fonds pour la promotion industrielle (FPI) à taux zéro ${ }^{18}$.

En outre, le Gouvernement a décidé de suspendre pour une période de 3 mois de certains contrôles fiscal, parafiscal et économique dans les entreprises suivi de la suspension durant 3 mois de l'application des pénalités en cas de retard dans le dédouanement de marchandises et produits de première nécessité. Cependant, toutes ces mesures n'avaient qu'une portée limitée dans la mesure où elles ne s'appliquaient qu'à certains produits dont la liste a été dressée par le ministre de l'économie. Le Gouvernement a décidé de l'orientation vers le guichet unique de toutes les perceptions douanières connexes qui s'effectuaient encore en dehors de ce guichet et la levée des barrières policières, postes de contrôle à l'intérieur du territoire sur les voies d'acheminement des produits de première nécessité ${ }^{19}$.

Le Gouvernement a suspendu pour une période de 3 mois la perception de la taxe sur la valeur ajoutée (TVA) sur l'importation et la vente de produits de première nécessité ou de consommation de masse. En ce qui concerne les politiques budgétaires, le Gouvernement devait améliorer « l'allocation de ressources par la notion de prééminence de dépenses à savoir la rémunération, priorités santé et relance sur toutes les dépenses, ainsi que la création d'un fond de soutien aux mesures de dépenses relatives à la crise ». Il devait également mobiliser les appuis budgétaires et ceux à la balance de paiement (Banque Mondiale $^{20}$, Banque africaine de Développement (BAD) ${ }^{21}$ et le Fonds Monétaire International (FMI) ${ }^{22}$.

17 Emilie Mboyo, «RDC : 1'Etat rend gratuite la fourniture d'eau et d'électricité aux ménages durant deux mois », disponible sur https://zoom-eco.net/a-la-une/rdc-letat-rend-gratuite, (Consulté le 02 juin 2020).

18 Emilie Mboyo, note 18.

19 Emilie Mboyo, note 18.

20 « La Banque mondiale alloue 47 millions de dollars à la République démocratique du Congo pour répondre à la pandémie de coronavirus » (Communiqué de presse 02 avril 2020), disponible sur https:/www.banquemondiale.org/fr/news/press-release/2020/04/02/the-world-bank-group-provide s-47, (Consulté le 25 juin 2020).

21 «Covid-19 : la BAD mobilise plus de 13 millions de dollars pour soutenir la zone CEMAC et la RD Congo », disponible sur https://www.agenceecofin.com/finances-publiques/0306-77192-covid -19, (Consulté le 25 juin 2020).

22 « Le FMI approuve un décaissement de 363,27 millions de dollars en faveur de la République démocratique du Congo pour aider le pays à faire face à la pandémie de COVID-19 », disponible sur https://www.imf.org/fr/News/Articles/2020/04/22/, (Consulté le 25 juin 2020). 
Cependant, ces mesures économiques n'ont pas concerné tous ceux qui exercent le petit commerce informel, plus particulièrement les femmes de la ville de Kinshasa. En réalité, les mesures économiques gouvernementales ont été édictées en méconnaissance totale de cette catégorie des femmes. Elles sont carrément exclues de mesures d'atténuation des effets néfastes de la COVID-19.

\section{Inefficacité du Fonds national de solidarité contre le coronavirus (FNSCC)}

Le Fonds national de solidarité contre le coronavirus est créé par l'Ordonnance n²0-018 du 06 avril 2020. Son organisation et son fonctionnement y sont régis. Le Fonds a pour principale mission de rechercher et collecter des moyens financiers destinés à servir, sous forme d'aides, assistance ou soutiens, aux personnes physiques ou morales, personnels médicaux soignants, services médicaux et hospitaliers ainsi qu'aux entreprises et autres structures exerçant une activité économique, qui seraient particulièrement touchés par les conséquences économiques, financières et sociales de la propagation du COVID-19 et des mesures prises pour en limiter la propagation ${ }^{23}$.

Se fondant sur l'article 5 de l'ordonnance qui donne les modalités de l'utilisation des ressources du fonds, l'on remarque que seules les entreprises en difficultés en raison de l'épidémie seront soutenues financièrement. Nulle part il est fait mention de soutien financier aux personnes physiques exerçant une activité économique, en l'occurrence les femmes exerçant le petit commerce dans l'informel, comme il est stipulé dans l'article $1^{\text {er }}$ de cette Ordonnance. Pourtant, elles figurent dans la catégorie des personnes victimes des conséquences économiques, financières et sociales des mesures d'urgences prises en vue de limiter la propagation du virus. Ce qui revient à dire que le FNSCC est ineffectif. La seule solution qui reste est le recours à la voie judiciaire.

\section{Nécessité de recourir à l'action judiciaire au titre de réparation du dommage exceptionnel}

Le dommage exceptionnel est défini comme étant celui qui résulte des mesures prises par les autorités publiques. L'article 89 de la Loi organique sur les juridictions de l'ordre administratif dispose :

« Dans les cas où il n'existe pas d'autre juridiction compétente, la section du contentieux du Conseil d'État connaît des demandes d'indemnités relatives à la réparation d'un dommage exceptionnel, matériel ou moral, résultant d'une mesure prise ou ordonnée par les autorités du pouvoir central, des provinces et des entités territoriales décentralisées ainsi que des organismes publics placés sous leur tutelle. Elle se pro-

23 Article de 1'Ordonnance $\mathrm{n}^{\circ} 20 / 018$ du 06 avril 2020 portant création, organisation et fonctionnement d'un Fonds National de Solidarité Contre le Coronavirus, en sigle «FNSCC », disponible sur http://www.leganet.cd, (Consulté le 01 décembre 2020). 
nonce en équité en tenant compte de toutes les circonstances d'intérêt public ou pri$v e^{24} »$.

La jurisprudence a dégagé les caractéristiques suivantes permettant de qualifier un dommage d'exceptionnel ${ }^{25}$ :

- Un dommage anormal, c'est-à-dire dépassant par sa nature ou son importance les gènes et les sacrifices courants qu'implique la vie en société;

- Un dommage direct résultant immédiatement de l'acte ou de l'abstention de l'autorité;

- Un dommage certain, c'est-à-dire ne dépendant pas d'une condition non encore réalisée et ne portant pas d'une condition non encore réalisée et ne portant pas sur des profits aléatoires ou des bénéfices espérés;

- Un dommage grave ce qui entraîne l'exclusion des simples gènes ou des préjudices mineurs;

- Un dommage spécial, c'est-à-dire n'atteignant pas toute une catégorie d'individus. Le préjudice doit n'avoir été subi que par quelques particuliers bien déterminés, voire par un seul d'entre eux.

Partant de ces éléments caractéristiques, il sied de noter que dans l'hypothèse de cette étude, il est vrai que le dommage est anormal dans ce sens qu'il va au-delà des sacrifices qu'implique la vie de ces femmes. Ce dommage résulte directement des mesures urgentes édictées par l'autorité qui est le Président de la République. Ce dommage est certain, ce qui revient à dire que ces femmes ne sont pas des potentielles victimes mais les sont en réalité. Il y a des cas palpables d'interview avec ces dernières attestant leurs situations actuelles misérables provoquées par les effets néfastes de la COVID-19. Enfin, ce dommage est spécial du fait que dans le cadre de cette étude, on ne démontre qu'une catégorie des femmes exerçant le petit commerce informel et atteintes négativement par ces mesures. De tout ce qui précède, il y a lieu de parler du dommage exceptionnel.

Le constituant congolais attribue au Conseil d'Etat la compétence de connaitre de la réparation du dommage exceptionnel en ces termes :

«Sans préjudice des autres compétences que lui reconnaît la Constitution ou la loi, le Conseil d'Etat connaît, dans les cas où il n'existe pas d'autres juridictions compétentes, de demandes d'indemnités relatives à la réparation d'un dommage exceptionnel, matériel ou moral résultant d'une mesure prise ou ordonnée par les autorités de

24 Art. 89 de la Loi organique du 15 octobre 2016 portant organisation, compétence et fonctionne-

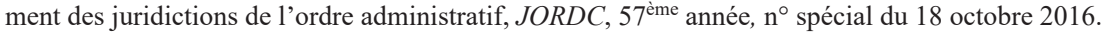

25 Barthélemy OMEONGA TONGOMO, Le contentieux de la réparation des préjudices exceptionnels résultant des actes des pouvoirs publics : Une étude des conditions de mise en jeu en droit comparé Belgique et RD Congo, Annales de la faculté de droit de l'Université de Kinshasa, Kinshasa, juin 2013, p. 207-208. 
la République. Il se prononce en équité en tenant compte de toutes les circonstances d'intérêt public ou privé26".

Suivant l'article 18 du Règlement intérieur du Conseil d'Etat, lorsque celui-ci statue en matière de demandes d'indemnités relatives à la réparation d'un dommage exceptionnel il s'assure que de l'inexistence, tant dans l'ordre administratif que dans l'ordre judiciaire, d'une juridiction compétente pour accorder pareille réparation, il examine le caractère exceptionnel du dommage en tenant compte des circonstances de fait et de droit ayant entouré la mesure ayant causé le dommage et en prenant en compte la gravité et la durée du préjudice subi. Le Conseil d'Etat doit statuer ex aequo et bono, sur les intérêts publics et privés des parties en présence, en cas de défaut de circonstances de fait et de droit d'appréciation du préjudice ${ }^{27}$.

Le Conseil d'Etat a déjà appliqué cette compétence dans l'arrêt RA.190²8 opposant la Société Africaine de Commerce Général et de Construction "SAFRICOM » Sarl (demanderesse en annulation) contre la RDC (défenderesse en annulation). Après examen de la requête, le Conseil d'Etat l'a déclarée recevable mais partiellement fondée. D'une part, le Conseil d'Etat répond favorablement à l'action de la partie demanderesse consistant en l'annulation des quatre arrêtés attaqués par celle-ci et; d'autre part, dit qu'il n'y a pas lieu aux dommages et intérêts au bénéfice de la victime (partie demanderesse) ayant constaté, en rapport avec le terrain visé, l'existence d'un arrêté ministériel n030 CAB/MIN.URB$\mathrm{HAB} / 2006$ du 09 décembre 2006 portant désaffectation du terrain du domaine public de l'Etat $^{29}$.

Se référant audit arrêt et se fondant aux dispositions constitutionnelles, il y a lieu de saisir le Conseil d'Etat en vue d'obtenir réparation et ce, selon une procédure spéciale. D'où l'intérêt de formuler quelques recommandations afin d'aider ces femmes afin qu'elles soient rétablies dans leur droit.

\section{CONCLUSION ET RECOMMANDATIONS}

Les mesures d'urgences prises par le Président de la République en vue de limiter la propagation de la COVID-19 ont bel et bien causé des effets négatifs sur les activités des femmes exercées dans l'informel dans la ville de Kinshasa. Après analyses de ces effets qui ont déséquilibré certains ménages, il a été important de penser aux solutions pour y remédier.

26 Article 155 al. 3 de la Constitution du 18 février 2006 telle que modifiée par la Loi $N^{\circ} 11 / 002$ du 20 janvier 2011 portant révision de certains articles de la République Démocratique du Congo, JORDC, $47^{\text {ème }}$ année, Numéro spécial, 18 février 2006.

27 Article 18 de l'ordonnance $\mathrm{n}^{\circ}$ 19/001 du 10 janvier 2019 portant Règlement intérieur du Conseil d'Etat, JORDC, 60 ${ }^{\mathrm{ème}}$ Année, Numéro spécial du 19 janvier 2019.

28 CE, 31 octobre 2019, RA.190, Requête en annulation de quatre requêtes ministériels, (inédit).

29 Note 11, onzième, douzième et treizième feuillet, inédit. 
Trois pistes de solutions ont été données. Les deux premières s'avèrent ne pas s'appliquer sur les activités de ces femmes de par le caractère informel de leurs activités. La seule solution est base de cette étude est celle de saisir le Conseil d'Etat pour réparation du dommage exceptionnel conformément à la législation en vigueur.

Ceci étant, les femmes victimes soutenues dans cette démarche obtiendront réparation du préjudice qu'elles ont subi surtout dans le contexte actuel du pays où il est dans la course de l'atteinte des ODD d'ici l'horizon 30 et signataires des autres textes juridiques en rapport avec l'autonomisation de la femme et l'égalité de genre.

Cette étude est une contribution à l'Etat de droit dans la mesure où elle milite pour la protection des droits économiques des personnes vulnérables qui sont les femmes. Pour ce faire, des recommandations sont formulées selon les étapes que doit suivre cette procédure. Dans la première étape, il sera question de procéder à l'identification de ces femmes victimes des mesures de l'état d'urgence. Cette identification pourra se faire par une descente sur terrain dans différentes communes ciblées de la ville de Kinshasa et interview afin de comprendre les attentes de ces femmes. Ceci permettra d'avoir un échantillon sur base duquel le reste du travail sera fait. Après cette identification, viendra l'étape d'organiser ces femmes. A l'issue des données récoltées sur terrain, il sera important de regrouper ces femmes en association professionnelle suivant la nature des activités exercées par chacune d'elles.

Enfin la dernière étape consistera à trouver un avocat. Celui-ci aura pour mission d'évaluer le préjudice subi et leur besoin réel, fixer le dommage et intérêt et saisir le Conseil d'Etat. A cet effet, le Conseil d'Etat prendra des décisions qui obligeront le Gouvernement à réparer les droits de ces personnes conformément aux dispositions légales y relatif.

\section{BIBLIOGRAPHIE}

\section{TEXTES JURIDIQUES}

- Constitution du 18 février 2006 telle que modifiée par la Loi $\mathrm{N}^{\circ} 11 / 002$ du 20 janvier 2011 portant révision de certains articles de la République Démocratique du Congo,

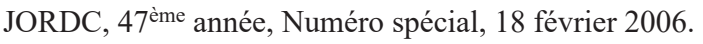

- Loi organique du 15 octobre 2016 portant organisation, compétence et fonctionnement des juridictions de l'ordre administratif, JORDC, $57^{\text {ème }}$ année, $\mathrm{n}^{\circ}$ spécial du 18 octobre 2016.

- ordonnance $\mathrm{n}^{\circ}$ 19/001 du 10 janvier 2019 portant Règlement intérieur du Conseil d'Etat, JORDC, 60 ème Année, Numéro spécial du 19 janvier 2019.

- Ordonnance n ${ }^{\circ}$ 20/014 du 24 mars 2020 portant proclamation de l'état d'urgence sanitaire pour faire face à l'épidémie de Covid-19, Journal Officiel de la République Démocratique du Congo (JORDC), 61 ${ }^{\text {ème }}$ année, Numéro spécial du 16 avril 2020. 
- Ordonnance $\mathrm{n}^{\circ} 20 / 018$ du 06 avril 2020 portant création, organisation et fonctionnement d'un Fonds National de Solidarité Contre le Coronavirus, en sigle « FNSCC », disponible sur http://www.leganet.cd, (Consulté le 01 décembre 2020).

\section{DOCTRINE}

\section{A. ARTICLES ET REVUES}

- Barthélemy OMEONGA TONGOMO, Le contentieux de la réparation des préjudices exceptionnels résultant des actes des pouvoirs publics : Une étude des conditions de mise en jeu en droit comparé Belgique et RD Congo, Annales de la faculté de droit de l'Université de Kinshasa, Editions Droits et Société « DES », Kinshasa, juin 2013, pp. 207-208.

- Genèse Bibi Ekomene, La promotion de l'entrepreneuriat féminin par le microcrédit en République Démocratique du Congo, Librairie africaine d'études juridiques 7 (2020), pp. 350-361.

- Genèse Bibi Ekomene, Renia Binaki Bamangana et Yvette Mutunda Tshibuila, Impact du covid-19 sur les femmes exerçant le petit commerce informel dans la ville de Kinshasa (RDC). Cas de madame Pauline Omoni vendeuse des fruits, Bananes plantains et arachides, disponible sur www.creeda-rdc.org.

- Genèse Bibi Ekomene, Renia Binaki Bamangana \&Yvette Mutunda Tshibuila, Impact du covid-19 sur les femmes exercant le petit commerce informel dans la ville de Kinshasa (RDC). Cas de madame claire $\mathrm{x}$, vendeuse de pains et arachides, https://www.cre eda-rdc.org/resources/documents/creeda-covid.pdf, (Consulté, le 30 janvier 2021).

- Genèse Bibi Ekomene, Renia Binaki Bamangana \&Yvette Mutunda Tshibuila,

- Impact du covid-19 sur les femmes exerçant le petit commerce informel dans la ville de Kinshasa (RDC). Cas de madame Mamie Luzayadio vendeuse de la farine de manioc, https://www.creeda-rdc.org/resources/documents/creeda-covid.pdf, (Consulté, le 30 janvier 2021).

- Juliette Dubois, « En RDC, l'activité tourne au ralenti et la pauvreté gagne du terrain à Kinshasa », https:/www.lemonde.fr/afrique/article/2020/10/29/, (Consulté le 30 janvier 2021). 


\section{JURISPRUDENCE}

- CE, 31 octobre 2019, RA.190, Requête en annulation de quatre requêtes ministériels, (inédit).

\section{AUTRES DOCUMENTS}

- Rapport du Gouvernement congolais intitulé «Impacts sanitaires et socioéconomiques de la COVID-19 en République Démocratique du Congo. Analyse prospective et orientations de la riposte multisectorielle », Kinshasa, mai 2020.

\section{SITES WEBOGRAPHIQUES}

- AFP \& TV5 Monde, « Coronavirus : ce que révèlent les taux de mortalité par pays et par continent », https://information.tv5monde.com/info/coronavirus-ce-que-revelent-les -taux-de-mortalite, (Consulté le 30 janvier 2021).

- https://www.oxfamfrance.org/inegalites-et-justice-fiscale/inegalites-et-pauvrete-en-fran ce-les-voyants-au-rouge/, (Consulté le 12 décembre 2020).

- https://www.jeuneafrique.com/915794/politique/coronavirus-en-rdc-felix-tshisekedi-de crete-letat-durgence-et-isole-kinshasa, (Consulté le 03 décembre 2020).

- Milaine Rossanaly, «À la découverte des femmes entrepreneurs de RDC : entre nécessité et ambition », https://www.banquemondiale.org/fr/news/feature/, (Consulté le 30 janvier 2021).

- Emilie Mboyo, «RDC : l'Etat rend gratuite la fourniture d'eau et d'électricité aux ménages durant deux mois », https://zoom-eco.net/a-la-une/rdc-letat-rend-gratuite, (Consulté le 02 juin 2020).

- «La Banque mondiale alloue 47 millions de dollars à la République démocratique du Congo pour répondre à la pandémie de coronavirus » (Communiqué de presse 02 avril 2020), https://www.banquemondiale.org/fr/news/press-release/2020/04/02/the-world-ba nk-group-provides-47, (Consulté le 25 juin 2020).

- «Covid-19 : la BAD mobilise plus de 13 millions de dollars pour soutenir la zone CEMAC et la RD Congo », https://www.agenceecofin.com/finances-publiques/0306-7719 2-covid-19, (Consulté le 25 juin 2020).

- «Le FMI approuve un décaissement de 363,27 millions de dollars en faveur de la République démocratique du Congo pour aider le pays à faire face à la pandémie de COVID-19 », https://www.imf.org/fr/News/Articles/2020/04/22/, (Consulté le 25 juin 2020). 